\title{
Nuclear shape phase transitions
}

\author{
D. VRETENAR
}

Physics Department, Faculty of Science, University of Zagreb, Zagreb, Croatia

\begin{abstract}
The evolution of shapes and shape (phase) transitions, including regions of short-lived exotic nuclei that are becoming accessible in experiments at radioactive-beam facilities, are governed by the shell structure of single-nucleon orbitals. In most cases the transition between different shapes is gradual but in a number of examples, with the addition or subtraction of only few nucleons, signatures of abrupt changes in observables are noticed. A quantitative analysis necessitates accurate modelling of the underlying nucleonic dynamics. Important advances have been reported in theoretical studies of complex shapes, especially in the "beyond mean-field" framework based on density functionals.
\end{abstract}

\section{Introduction and theory framework}

The evolution of equilibrium shapes and the corresponding excitation dynamics present some of the most studied low-energy nuclear phenomena, both experimentally and theoretically [1-4]. Different shapes, shape coexistence, and shape transitions take place in light, medium-heavy, and heavy nuclei, and have their origin in the evolution of single-nucleon shell structure that reflects the underlying confining single-particle potential. In contrast to spherical shapes that occur for the particular case of single or doubly closed-shell nuclei, deformed equilibrium shapes arise due to strong protonneutron correlations in open-shell nuclei. Far from the $\beta$-stability line, in particular, the energy spacings between single-particle levels can be very different from those found in stable nuclei. This can result in reduced spherical 
shell gaps, modifications of magic numbers, occurrence of islands of inversion, deformed equilibrium shapes and coexistence of shapes with different deformations, and shape (phase) transitions.

As the number of nucleons changes from nucleus to nucleus, in general one observes a gradual evolution of different shapes - spherical, axially quadrupole deformed, shapes that are soft with respect to triaxial deformations, octupole shapes $[1,2]$. In a number of cases the addition or subtraction of only a few nucleons leads to rapid changes in equilibrium shapes and shape phase transitions may occur [4]. Phase transitions in the equilibrium shapes of nuclei correspond to first- and second-order quantum phase transitions (QPTs) between competing ground-state phases induced by variation of a non-thermal control parameter at zero temperature. Particularly important are studies of observables that can be related to order parameters and the degree to which discontinuities at a phase transitional point are smoothed out in finite nuclei. In recent years nuclear QPTs have been investigated extensively, both in experimental studies and employing a variety of theoretical models. An integral microscopic description of these universal phenomena must be based on a theoretical framework that can be extended from light to heavy nuclei, and from the line of $\beta$-stability to the nucleon drip lines. Such a framework is the one based on nuclear density functional theory (DFT).

Nuclear energy density functionals (EDFs) enable a global and accurate microscopic description of ground-state properties and collective excitations over the entire nuclide chart. The basic implementation of the EDF framework is in terms of self-consistent mean-field (SCMF) models, in which an EDF is constructed as a functional of one-body nucleon densities that correspond to a single product state. This approach to nuclear structure is analogous to Kohn-Sham DFT. Nuclear SCMF models effectively map the many-body problem onto a one-body problem, and the exact EDF is approximated by functionals of powers and gradients of ground-state nucleon densities and currents $[5,6]$. When considering applications that go beyond the simple mean-field level, that is, to calculate excitation spectra and electromagnetic transition rates in individual nuclei, it is necessary to extend the Kohn-Sham EDF framework to include correlations that arise from symmetry restoration and fluctuations around the mean-field minimum [5-7]. The second level of implementation of nuclear EDFs takes into account collective correlations through the restoration of broken symmetries and configuration mixing of symmetry-breaking product states. In this work we consider two examples of applications of structure models based on relativistic EDFs in studies of complex shapes, shape transitions and the corresponding excitation spectra and decay patterns. 
Over the last two decades a number of successful relativistic mean-field (RMF) models have been constructed based on the framework of quantum hadrodynamics, in which a nucleus is described as a system of Dirac nucleons coupled to exchange mesons through an effective Lagrangian [8]. However, at energies characteristic for nuclear structure phenomena, meson exchange $(\sigma, \omega, \rho, \ldots)$ is just a convenient representation of the effective inter-nucleon interaction. The exchange of heavy mesons is not resolved at low energies, and can be replaced by the corresponding local contact interactions between nucleons.

The basic building blocks of a relativistic nuclear energy density functional are the densities and currents bilinear in the Dirac spinor field $\psi$ of the nucleon: $\bar{\psi} \mathcal{O}_{\tau} \Gamma \psi, \mathcal{O}_{\tau} \in\left\{1, \tau_{i}\right\}, \Gamma \in\left\{1, \gamma_{\mu}, \gamma_{5}, \gamma_{5} \gamma_{\mu}, \sigma_{\mu \nu}\right\}$. Here $\tau_{i}$ are the isospin Pauli matrices. The nuclear ground-state density and energy are determined by the self-consistent solution of relativistic linear single-nucleon Kohn-Sham equations. To derive those equations it is useful to construct an interaction Lagrangian with contact interaction terms in the various isospace-space channels: isoscalar-scalar $(\bar{\psi} \psi)^{2}$, isoscalarvector $\left(\bar{\psi} \gamma_{\mu} \psi\right)\left(\bar{\psi} \gamma^{\mu} \psi\right)$, isovector-scalar $(\bar{\psi} \vec{\tau} \psi) \cdot(\bar{\psi} \vec{\tau} \psi)$, and isovector-vector $\left(\bar{\psi} \vec{\tau} \gamma_{\mu} \psi\right) \cdot\left(\bar{\psi} \vec{\tau} \gamma^{\mu} \psi\right)$. A general Lagrangian can be written as a power series in the currents $\bar{\psi} \mathcal{O}_{\tau} \Gamma \psi$ and their derivatives, with higher-order terms representing in-medium many-body correlations. Alternatively, one constructs a Lagrangian that comprise second-order interaction terms only, with manybody correlations encoded in density-dependent coupling functions. This type of RMF-based models has been successfully used in analyses of a variety of structure phenomena, not only in nuclei along the valley of $\beta$-stability, but also in exotic nuclei with extreme isospin values and close to the particle drip lines. In the present study illustrative calculations are performed with the EDF DD-PC1 [9]. Starting from microscopic nucleon self-energies in nuclear matter, the density-dependent coupling parameters of the DDPC1 point-coupling Lagrangian were fine-tuned to the experimental masses of a set of 64 deformed nuclei in the mass regions $A \approx 150-180$ and $A \approx 230-250$. The functional has been further tested in a number of mean-field and beyond-mean-field calculations in different mass regions. For the examples considered here, pairing correlations are taken into account by employing an interaction that is separable in momentum space, and is completely determined by two parameters adjusted to reproduce the empirical bell-shaped pairing gap in symmetric nuclear matter [10].

The analysis of ground-state properties and low-energy collective excitations starts with a calculation of deformation energy surfaces with mass multipole moments as constrained quantities. The static nuclear mean-field 
is characterised by the breaking of symmetries of the underlying Hamiltonian - translational, rotational, particle number. To calculate spectroscopic properties it is necessary to extend the SCMF scheme to include collective correlations that arise from symmetry restoration and fluctuations around the mean-field minima. Many studies of the evolution of low-energy collective states have been carried out by employing the Generator Coordinate Method (GCM) in configuration mixing calculations of angular-momentum and particle-number projected states based on relativistic energy density functionals $[11,12]$. The application of this method to medium-heavy and heavy nuclei presents a more involved and technically difficult problem and, therefore, only few studies of collective states in heavier systems have been reported.

In an alternative approach a collective Hamiltonian can be formulated, with deformation-dependent parameters determined by self-consistent mean-field calculations. The dynamics of the collective Hamiltonian is governed by the vibrational inertial functions and the moments of inertia, and these functions are determined by the energy density functional and the effective pairing interaction [6]. For instance, in the case of quadrupole degrees of freedom:

$$
\hat{H}_{\text {coll }}=\hat{T}_{\text {vib }}+\hat{T}_{\text {rot }}+V_{\text {coll }},
$$

where the vibrational kinetic energy is parameterized by the the mass parameters $B_{\beta \beta}, B_{\beta \gamma}, B_{\gamma \gamma}$, the three moments of inertia $\mathcal{I}_{k}$ determine the rotational kinetic energy, and $V_{\text {coll }}$ is the collective potential. The self-consistent mean-field solution for the single-quasiparticle energies and wave functions for the entire energy surface, as functions of the quadrupole deformations $\beta$ and $\gamma$, provide the microscopic input for calculation of the mass parameters, moments of inertia and the collective potential. The Hamiltonian describes quadrupole vibrations, rotations, and the coupling of these collective modes. The diagonalization gives the excitation energies and collective wave functions that can be used to calculate various observables. The details of the particular implementation of the relativistic EDF-based collective Hamiltonian used in the present study can be found in Ref. [6].

\section{Triaxial shapes in germanium isotopes}

Most deformed nuclei display axially-symmetric quadrupole equilibrium shapes but in a number of cases axial symmetry is explicitly broken. Triaxial quadrupole shapes can be described in terms of two polar deformation parameters: $\beta$ (proportional to the intrinsic quadrupole moment) and the 

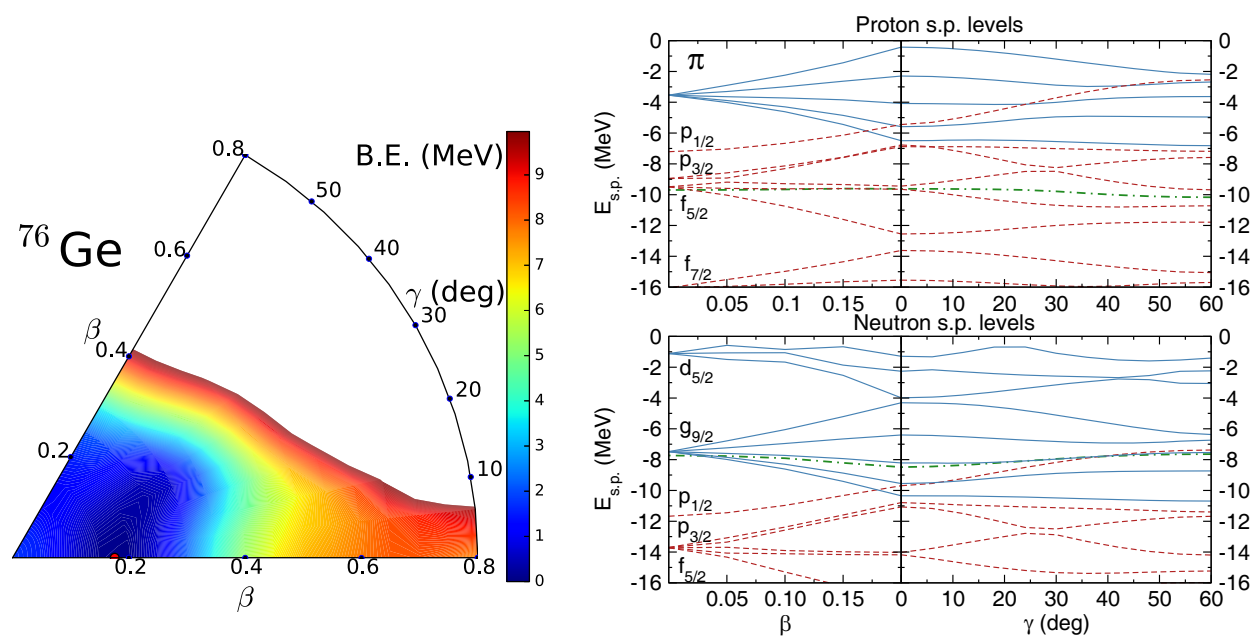

Figure 1: Left: Self-consistent RHB deformation energy surface of ${ }^{76} \mathrm{Ge}$ in the $\beta-\gamma$ plane $\left(0 \leq \gamma \leq 60^{0}\right)$. Right: Proton and neutron energy levels of ${ }^{76} \mathrm{Ge}$ as functions of the deformation parameters.

angular variable $\gamma$. Two simple geometrical models describe the limiting cases of a triaxial shape: in the rigid-triaxial rotor model the collective potential has a stable minimum at a particular value of $\gamma(\gamma$-rigid potential), whereas in the $\gamma$-unstable rotor model the potential is independent of $\gamma$ ( $\gamma$-soft potential). A recent analysis [13] based on nuclear density functional theory, has shown that neither the limit of rigid-triaxial rotor nor the the $\gamma$-unstable rotor are actually realised in finite nuclei. The systematics of low-lying collective spectra and transition rates indicate that non-axial medium-heavy and heavy nuclei lie almost exactly in the middle between the two geometrical limits.

Empirical evidence for rigid triaxial deformation at low energy in ${ }^{76} \mathrm{Ge}$ was recently reported in [14], where it was shown that the phase of the odd-even staggering of the $\gamma$-band is consistent with the assumption of a $\gamma$-rigid structure, although the amplitude of the staggering is considerably smaller than that predicted by the rigid-triaxial rotor model. In the left panel of Fig. 1 we thus plot the triaxial quadrupole energy map of ${ }^{76} \mathrm{Ge}$ in the $\beta-\gamma$ plane $\left(0 \leq \gamma \leq 60^{0}\right)$. The deformation energy surface displays an axially-symmetric minimum on the prolate axis, but one also notices a considerable softness in the $\gamma$ direction. Mean-field shapes are governed by the evolution of the underlying shell structure of single-nucleon orbitals, and the formation of deformed minima is related to the occurrence of regions of 

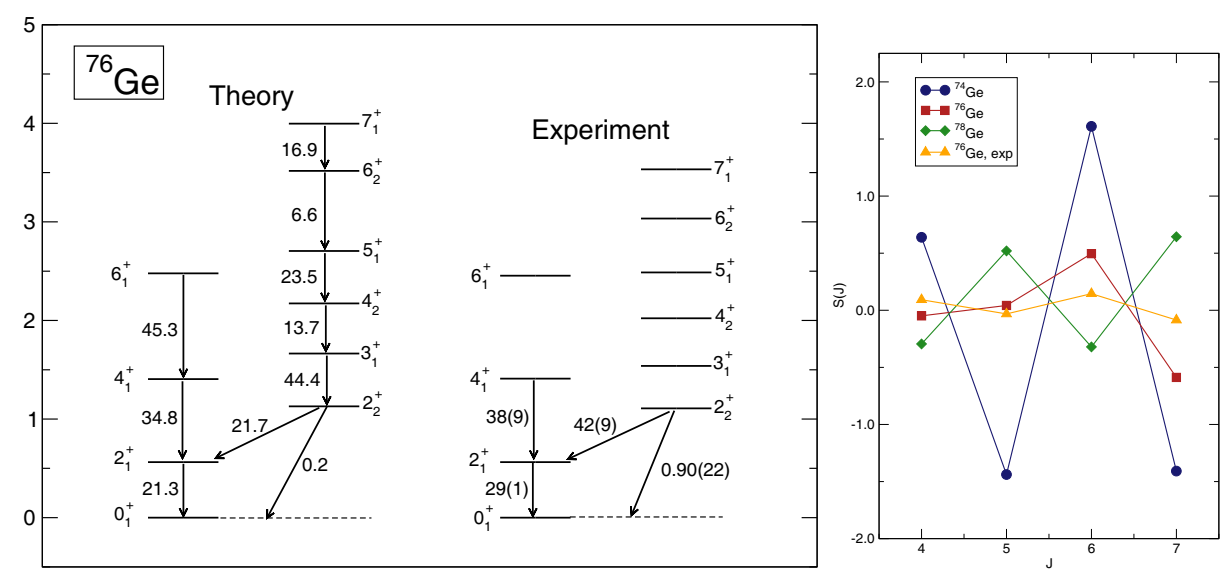

Figure 2: Left: The excitation spectrum of ${ }^{76} \mathrm{Ge}$ calculated with the collective Hamiltonian and compared to data. $B(E 2)$ values are in Weisskopf units. Right: The staggering $S(J)$ in the $\gamma$-bands of ${ }^{74,76,78} \mathrm{Ge}$ isotopes.

low single-particle level density around the Fermi surface. The right panel of Fig. 1 displays the proton and neutron single-particle energy levels in ${ }^{76} \mathrm{Ge}$. Solid (blue) and dashed (red) curves correspond to levels with positive and negative parity, respectively. The dot-dashed (green) curves denote the Fermi level. The single-particle levels are plotted as functions of the deformation parameters along a path in the $\beta-\gamma$ plane. Starting from the spherical configuration, we follow the single-nucleon levels on a path along the prolate axis up to the approximate position of the minimum and then, for this fixed value of $\beta$, we trace the levels along the path from $\gamma=0^{0}$ to $\gamma=60^{0}$. Proton levels show a pronounced gap between the last occupied and first unoccupied levels in the triaxial region at $\gamma \approx 30^{\circ}$, whereas for the neutron levels the gap appears to be almost independent of $\gamma$. Because of small energy differences between different mean-field configurations, dynamical effects related to restoration of broken symmetries and fluctuations in collective coordinates can have a decisive role in the eventual formation of a triaxial ground state [15].

Figure 2 displays the spectrum of ${ }^{76} \mathrm{Ge}$ calculated with the collective Hamiltonian based on the functional DD-PC1. Levels that belong to the ground-state band and the $\gamma$-band are compared to the experimental spectrum [14], and assigned either to the ground-state band or the $\gamma$-band according to the distribution of the projection $K$ of the angular momentum $J$ on the $z$ axis in the body-fixed frame. Yrast states have a predominant $K=0$ component in the wave functions, whereas the $\gamma$-band comprises 
states above the yrast characterized by dominant $K=2$ components. The level of $K$-mixing is reflected in the staggering in energy between odd- and even-spin states in the $\gamma$-band, and it can be quantified by considering the differential quantity:

$$
S(J)=\frac{E\left[J_{\gamma}^{+}\right]-2 E\left[(J-1)_{\gamma}^{+}\right]+E\left[(J-2)_{\gamma}^{+}\right]}{E\left[2_{1}^{+}\right]} .
$$

For a deformed $\gamma$-soft potential, $S(J)$ has negative values for even-spin states and positive values for odd-spin states, with the magnitude slowly increasing with spin. For a triaxial potential the level clustering in the $\gamma$-band is opposite, and $S(J)$ oscillates between positive values for even-spin states and negative values for odd-spin states. In the right panel of Fig. 2 we plot the calculated values for the staggering $S(J)$ in the $\gamma$-bands of ${ }^{74,76,78} \mathrm{Ge}$, and the experimental values for ${ }^{76} \mathrm{Ge}$ [14]. The phase of $S(J)$ for ${ }^{74} \mathrm{Ge}$ is consistent with a $\gamma$-rigid triaxial shape, whereas ${ }^{78} \mathrm{Ge}$ displays the opposite pattern for $S(J)$, that is, the $\gamma$-band indicates a soft shapes. ${ }^{76} \mathrm{Ge}$ is at the (shape) transition between the triaxial ${ }^{74} \mathrm{Ge}$ and the $\gamma$-soft heavier isotopes. For this nucleus the amplitudes of $S(4)$ and $S(5)$ almost vanish, whereas $S(6)$ and $S(7)$ follow a pattern characteristic for triaxial shapes, but with considerably smaller amplitudes. The theoretical results are in very good agreement with available data, both for the ratio $E\left(2_{2}^{+}\right) / E\left(2_{1}^{+}\right)=2$ and the pattern and amplitude of the staggering $S(J)$. The self-consistent meanfield calculation has shown that the energy surface of ${ }^{76} \mathrm{Ge}$ is $\gamma$-soft. The inclusion of collective correlations drives the nucleus toward triaxiality, but the correlations are not strong enough to stabilise a $\gamma \approx 30^{\circ}$ triaxial shape.

\section{Octupole shape-phase transitions in Thorium}

Reflection-asymmetric shapes are characterized by the presence of negativeparity bands, and by pronounced electric dipole and octupole transitions. In the case of static octupole deformation the lowest $\pi=+1$ even-spin states and the $\pi=-1$ odd-spin states form an alternating-parity band. Octupole deformations develop through a coupling of orbitals in the vicinity of the Fermi surface with quantum numbers $(l, j)$ and an intruder unique-parity orbital with $(l+3, j+3)[2]$. For instance, in the case of the $(N \approx 134$ and $Z \approx 88$ ) nuclei in the region of light actinides, the coupling of the neutron orbitals $g_{9 / 2}$ and $j_{15 / 2}$, and that of the proton single-particle states $f_{7 / 2}$ and $i_{13 / 2}$, can result in octupole mean-field deformations.

In light actinides an interesting structure phenomenon is the simultaneous transition between quadrupole equilibrium shapes, and from stable 

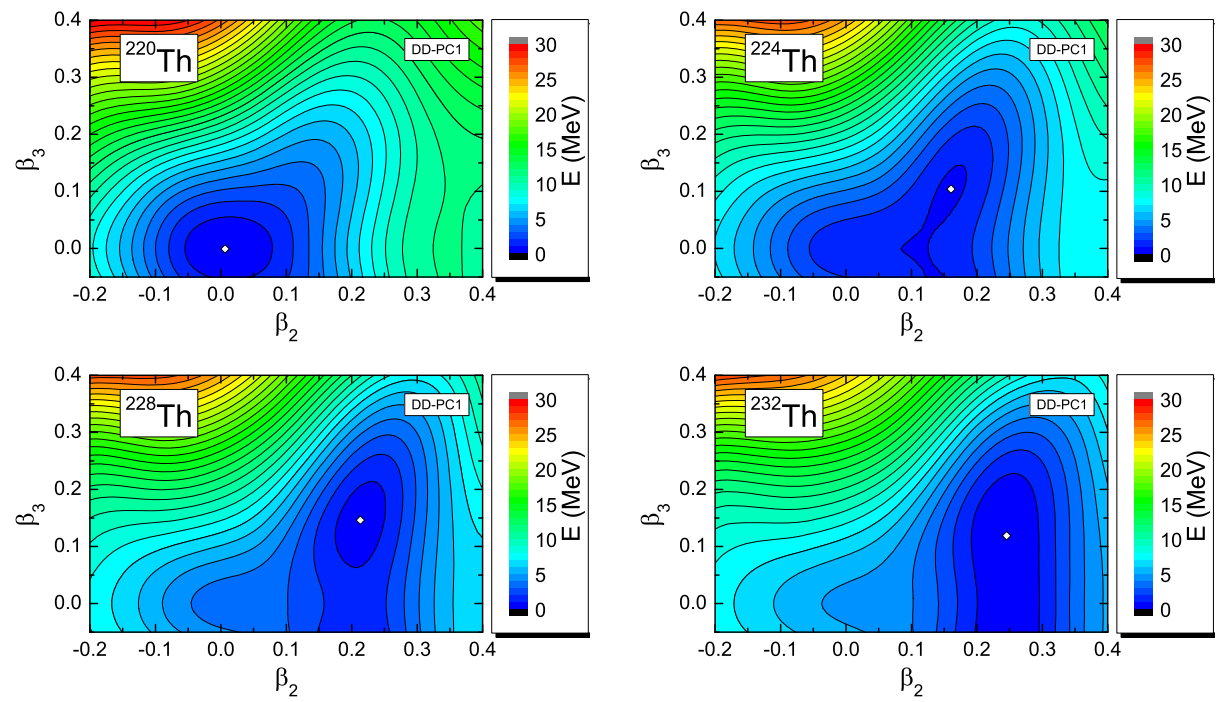

Figure 3: Microscopic DD-PC1 self-consistent axially symmetric energy surfaces of the nuclei ${ }^{220-232} \mathrm{Th}$ in the $\left(\beta_{2}, \beta_{3}\right)$ plane, symmetric with respect to $\beta_{3}=0$ axis. The contours join points on the surface with the same energy, and the separation between neighbouring contours is $1 \mathrm{MeV}$.

octupole deformations to octupole vibrations [16-18]. In Fig. 3 we plot the axially symmetric deformation energy surfaces in the $\left(\beta_{2}, \beta_{3}\right)$ plane for the isotopes ${ }^{220-232} \mathrm{Th}$, calculated with the functional DD-PC1. A soft spherical energy surface is predicted for ${ }^{220,222} \mathrm{Th}$. The quadrupole deformation becomes more pronounced in ${ }^{224} \mathrm{Th}$ and, in addition, octupole deformation develops. The energy minimum is found in the $\beta_{3} \neq 0$ region, located at $\left(\beta_{2}, \beta_{3}\right) \approx(0.15,0.1)$. From ${ }^{224} \mathrm{Th}$ to ${ }^{228} \mathrm{Th}$ a distinct octupole minimum is calculated whereas, starting from ${ }^{228} \mathrm{Th}$, the minimum becomes softer in $\beta_{3}$ direction. Soft octupole surfaces are predicted for ${ }^{230,232} \mathrm{Th}$.

A quadrupole-octupole collective Hamiltonian [17] can be used to analyse simultaneous quadrupole and octupole shape transitions. An alternative and more economic approach is the interacting-boson model (IBM), based on the EDF framework $[16,18]$. The $s d f$ IBM Hamiltonian is constructed by mapping the microscopic quadrupole-octupole fermion energy surface onto the equivalent one in the corresponding boson system. Figure 4 displays the IBM excitation spectra of the ground-state band $\left(K^{\pi}=0_{1}^{+}\right)$and the lowest negative-parity band $\left(K^{\pi}=0_{1}^{-}\right)$in ${ }^{220-232} \mathrm{Th}$, obtained starting from the DD-PC1 constrained self-consistent mean-field solutions (cf. Fig. 3). The energies of the $\pi=+1$ levels systematically decrease in energy with mass 


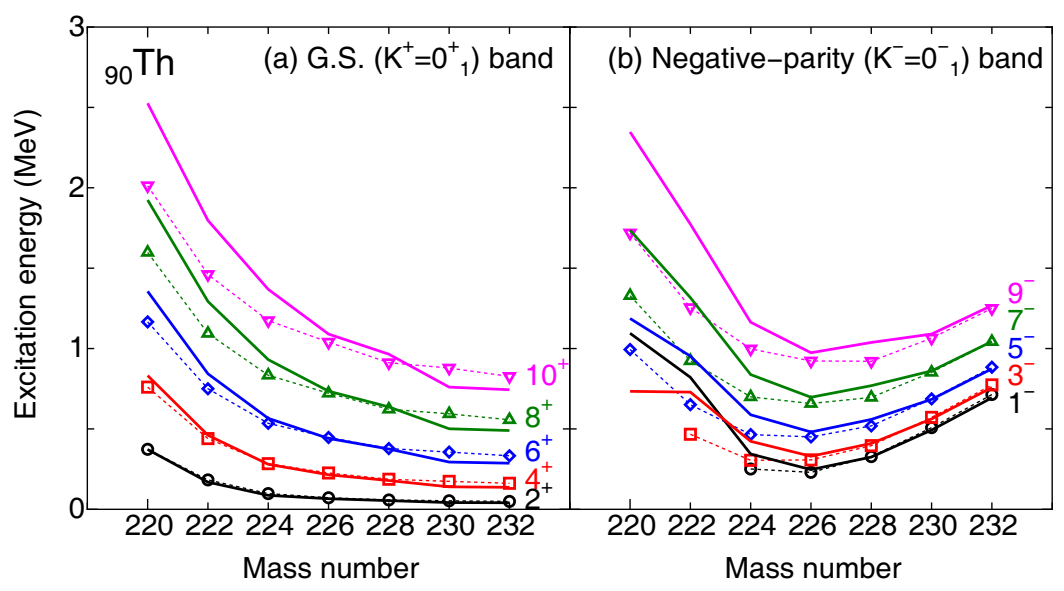

Figure 4: Isotopic dependence of the excitation energies of levels of the positiveparity ground-state band $\left(K^{\pi}=0_{1}^{+}\right)(\mathrm{a})$, and the lowest negative-parity band $\left(K^{\pi}=0_{1}^{-}\right)(\mathrm{b})$, for ${ }^{220-232} \mathrm{Th}$. In each panel solid lines and symbols denote the theoretical and experimental values, respectively.

number, reflecting the increase of quadrupole collectivity. ${ }^{220,222} \mathrm{Th}$ exhibit a quadrupole vibrational structure, while ground-state rotational bands are obtained in ${ }^{226-232} \mathrm{Th}$. The $\pi=-1$ bands form a parabolic structure centred at ${ }^{226} \mathrm{Th}$, in which the octupole deformed minimum is most pronounced. Starting from ${ }^{226} \mathrm{Th}$, the energies of negative-parity states systematically increase and the band becomes more compressed. A rotational-like collective band based on the octupole vibrational state $1_{1}^{-}$develops.

The vertex of the parabola of the $\pi=-1$ states can be associated with a phase transition between stable octupole deformations and octupole vibrations characteristic for $\beta_{3}$-soft potentials, with the excitation energy of the $1_{1}^{-}$band-head as the order parameter. Another signature of the phase transition is the odd-even staggering $E(J) / E\left(2_{1}^{+}\right)$. In lighter Th isotopes the staggering is negligible, and it only becomes more pronounced from ${ }^{228} \mathrm{Th}$, indicating that negative-parity states form a separate rotational band built on the octupole vibration. Thorium isotopes therefore exhibit a transition from spherical shapes near ${ }^{220} \mathrm{Th}$ to stable octupole and quadrupole deformations around ${ }^{226} \mathrm{Th}$, and the development of octupole vibrations characteristic for $\beta_{3}$-soft potentials in heavier Th nuclei, with the occurrence of a shape phase transition near ${ }^{226} \mathrm{Th}$. 


\section{References}

[1] Heyde K. and Wood J. L., Rev. Mod. Phys., 83 (2011) 1467.

[2] Butler P. A. and Nazarewicz W., Rev. Mod. Phys., 68 (1996) 349.

[3] Sorlin O. and Porquet M.-G., Prog. Part. Nucl. Phys., 61 (2008) 602.

[4] Cejnar P., Jolie J., and Casten R. F., Rev. Mod. Phys., 82 (2010) 2155.

[5] Bender M., Heenen P.-H., and Reinhard P.-G., Rev. Mod. Phys., 75 (2003) 121.

[6] Nikšić T., Vretenar D., and Ring P., Prog. Part. Nucl. Phys., 66 (2011) 519.

[7] Duguet T., in Lecture Notes in Physics, 879 (Springer, Heidelberg) 2014, pp. 293-350.

[8] Vretenar D., Afanasjev A.V., Lalazissis G.A., and Ring P., Phys. Rep., 409 (2005) 101.

[9] Nikšić T., Vretenar D., and Ring P., Phys. Rev. C, 78 (2008) 034318.

[10] Tian Y., Ma Z. Y., and Ring P., Phys. Lett. B, 676 (2009) 44.

[11] Yao J. M., Meng J., Ring P., and Vretenar D., Phys. Rev. C, 81 (2010) 044311.

[12] Yao J. M., Mei H., Chen H., Meng J., Ring P., and Vretenar D., Phys. Rev. C, 83 (2011) 014308.

[13] Nomura K., Shimizu N., Vretenar D., Nikšić T., and Otsuka T., Phys. Rev. Lett., 108 (2012) 132501.

[14] Toh Y. et al., Phys. Rev. C, 87 (2013) 041304(R).

[15] Nikšić T., Marević P., and Vretenar D., Phys. Rev. C, 89 (2014) 044325.

[16] Nomura K., Vretenar D., and Lu B.-N., Phys. Rev. C, 88 (2013) $021303(\mathrm{R})$.

[17] Li Z. P., Song B. Y., Yao J. M., Vretenar D., and Meng J., Phys. Lett. $B$ 726, (2013) 866 .

[18] Nomura K., Vretenar D., Nikšić T., and Lu B.-N., Phys. Rev. C, 89, (2014) 024312. 